\title{
CABYR binds to AKAP3 and Ropporin in the human sperm fibrous sheath
}

\author{
Yan-Feng Li ${ }^{1}$, Wei $\mathrm{He}^{2}$, Arabinda Mandal ${ }^{3}$, Young-Hwan Kim ${ }^{3}$, Laura Digilio ${ }^{3}$, Ken Klotz ${ }^{3}$, Charles J Flickinger ${ }^{3}$ \\ and John C Herr ${ }^{3}$
}

Calcium-binding tyrosine phosphorylation-regulated protein (CABYR) is a highly polymorphic calcium-binding tyrosine- and serine-/threonine-phosphorylated fibrous sheath (FS) protein involved in capacitation. A putative domain (amino acids 12-48) homologous to the regulatory subunit of type II CAMP-dependent protein kinase A (RII) dimerisation and A kinase-anchoring protein (AKAP)-binding domains of protein kinase $\mathrm{A}$ at the $\mathrm{N}$-terminus suggests that CABYR may self-assemble and bind to AKAPs. Moreover, there is evidence that CABYR has limited interaction with AKAPs. However, further evidence and new relationships between CABYR and other FS proteins, including AKAPs, will be helpful in understanding the basic physiology of FS. In this study, a new strategy for co-immunoprecipitation of insoluble proteins, as well as the standard co-immunoprecipitation method in combination with mass spectrometry and western blot, was employed to explore the relationship between CABYR, AKAP3 and Ropporin. The results showed that AKAP3 was co-immunoprecipitated with CABYR by the anti-CABYR-A polyclonal antibody, and, conversely, CABYR was also co-immunoprecipitated with AKAP3 by the anti-AKAP3 polyclonal antibody. Another RII-like domain containing protein, Ropporin, was also co-immunoprecipitated with CABYR, indicating that Ropporin is one of CABYR's binding partners. The interactions between CABYR, AKAP3 and Ropporin were confirmed by yeast two-hybrid assays. Further analysis showed that CABYR not only binds to AKAP3 by its RII domain but binds to Ropporin through other regions besides the RII-like domain. This is the first demonstration that CABYR variants form a complex not only with the scaffolding protein AKAP3 but also with another RII-like domain-containing protein in the human sperm FS. Asian Journal of Andrology (2011) 13, 266-274; doi:10.1038/aja.2010.149; published online 17 January 2011

Keywords: AKAP3; CABYR; fibrous sheath; Ropporin; sperm tail; spermatozoa; Western blotting

\section{INTRODUCTION}

The intact sperm flagellum has four distinct segments: the connecting piece adjacent to the head, the middle piece defined by a tightly packed helical array of mitochondria surrounding the cytoskeletal structures of the flagellum, the principal piece, which constitutes about three quarters of the length of the flagellum and is enclosed by the fibrous sheath (FS), and the short end piece. ${ }^{1}$ The FS is a unique cytoskeletal structure that underlies the plasma membrane, surrounds the axoneme and outer dense fibres, and defines the extent of the principal piece region of the sperm flagellum. It consists of two longitudinal columns connected by closely arrayed circumferential ribs. The traditional opinion about the FS is that it provides mechanical support for the sperm tail, influences the degree of flexibility by modulating flagellar bending, and defines the plane of flagellar motion and the shape of the flagellar beat. ${ }^{1,2}$ However, proteins associated with the FS identified in recent studies indicate that it serves as a scaffold for both glycolytic enzymes and constituents of signalling cascades and plays a role in the regulation of sperm motility. ${ }^{1}$

More than 20 proteins that are located in or are closely related to the FS of mammalian spermatozoa have been reported. They include A kinaseanchoring protein 3 (AKAP3), ${ }^{3,4}$ AKAP4, ${ }^{5,6}$ testis-specific, developmentally regulated A-kinase-anchoring protein-80 (TAKAP-80), ${ }^{7}$ glyceraldehyde phosphate dehydrogenase-S (GAPDS), ${ }^{8,9}$ type 1 hexoki-

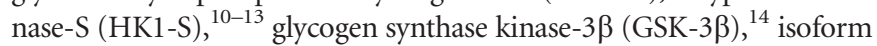
of aldolase 1 (ALDOA), lactate dehydrogenase-A (LDH-A), ${ }^{15}$ sperm flagellar energy carrier (SFEC), triose phosphate isomerase, glyceraldehyde 3-phosphate dehydrogenase (GAPDH), pyruvate kinase, lactate dehydrogenase-C (LDH-C), sorbitol dehydrogenase, ${ }^{16}$ glutathione S-transferase mu 5 (GSTM5), ${ }^{17}$ FS39, ${ }^{18}$ Ropporin, Rhophilin, ${ }^{19}$ sperm autoantigenic protein 17 (SP-17), ${ }^{20-22}$ phosphodiesterase 4A (PDE4A), ${ }^{23}$ fibrous sheath interacting protein 1 (FSIP1), fibrous sheath interacting protein 2 (FSIP2), ${ }^{6}$ AKAP-associated sperm protein (ASP), ${ }^{22}$ fibrous sheath calcium-binding tyrosine phosphorylation-regulated protein (CABYR)-binding protein (FSCB) ${ }^{24}$ and CABYR. ${ }^{25}$ Very little is known about how the proteins are assembled into the complex unique structure of the FS and how they bind to each other in the FS.

CABYR was initially found by the identification of calcium-binding and proteins phosphorylated by tyrosine kinases using two-dimensional (2D) gel analysis based on a proteomic strategy to identify targets at the intersection of the calcium and protein tyrosine kinase signal transduction pathways in human spermatozoa. ${ }^{25}$ It is a highly polymorphic, calcium-binding protein that is phosphorylated on tyrosine $e^{25}$ as well as serine or threonine ${ }^{26}$ during capacitation. Six splice 
variants of human CABYR have been reported and involve two coding regions, coding region $\mathrm{A}$ and $\mathrm{B}$ of CABYR gene (CR-A and CR-B). CABYR possesses putative motifs for self-assembly and for binding to AKAP. ${ }^{25} \mathrm{~N}$-terminal amino acids $12-48$ of human CABYR (accession no. AF088868) bear a $40 \%$ identity and 59\% similarity to amino acids 8-44 of human regulatory subunit of type II alpha cAMP-dependent protein kinase A (RII $\alpha)$. The N-terminal amino acids 12-44 of human Ropporin (accession no. XP_945818) bear a 33\% identity and 61\% similarity to amino acids $8-44$ of human RII $\alpha$. Although some evidence has shown that CABYR and Ropporin bind AKAPs, ${ }^{22,27-29}$ looking for further evidence and new interactions between CABYR and other FS proteins, including AKAPs and Ropporin, will be important for understanding the basic physiology of the FS.

\section{MATERIALS AND METHODS}

\section{Antibodies and reagents}

Human spermatozoa were obtained from student volunteers at the University of Virginia, Charlottesville, VA, USA. Rat polyclonal antihuman CABYR-A (the protein expressed by CR-A), anti-human CABYR-B (the protein expressed by CR-B), and anti-human AKAP serum were prepared by our research group at the University of Virginia, and their specificity has been demonstrated previously., 4,25,30,31 Horseradish peroxidase-conjugated goat anti-rat immunoglobulin $\mathrm{G}$ was purchased from Sigma-Aldrich (St Louis, MO, USA). An immunoprecipitation kit was purchased from Roche Applied Science (Indianapolis, IN, USA). IPG strips and Criterion 4-15\% linear gradient gels were purchased from Bio-Rad (Hercules, CA, USA). Dialysis cassettes were purchased from Pierce (Rockford, IL, USA). Ampholines were purchased from Amersham Pharmacia Biotech (Piscataway, NJ, USA). Human testicular marathon-ready cDNA was purchased from BD BiosciencesClontech (San Jose, CA, USA). The Matchmaker Gal4 Two-Hybrid System 3 was purchased from Clontech (Palo Alto, CA. USA).

\section{Co-immunoprecipitation of human sperm proteins by polyclonal anti-CABYR-A or anti-AKAP3 serum}

To optimize co-immunoprecipitation of CABYR and AKAP3 from human spermatozoa, two different methods were used: (i) method 1: semen specimens were obtained by masturbation from normal, healthy young men. Only ejaculates with normal values of semen parameters defined by WHO in $1992^{32}$ were used in this study. Individual semen samples were allowed to liquefy at room temperature (normally for $1 \mathrm{~h}$, range: $0.5-3 \mathrm{~h}$ ). The motile spermatozoa were harvested by the swim-up method of Bronson and Fusi. ${ }^{33}$ Immunoprecipitations were performed on $2 \times 10^{8}$ spermatozoa and $20 \mu \mathrm{l}$ of rat polyclonal anti-CABYR-A or pre-immune serum following the manufacturer's instructions. The protein $\mathrm{G}$-agarose was pelleted at $12000 \mathrm{~g}$ for $20 \mathrm{~s}$ at room temperature. Immune complexes were dissociated in $200 \mu$ l Celis buffer $\left(9.8 \mathrm{~mol}^{-1}\right.$ urea, $2 \%(\mathrm{v} / \mathrm{v})$ Nonidet P-40, $100 \mathrm{mmol} \mathrm{l}^{-1}$ dithiothreitol (DTT) with a protease inhibitor mixture (Roche Applied Science) at $4{ }^{\circ} \mathrm{C}$ for 20 min with gentle shaking and then separated by $2 \mathrm{D}$ gel electrophoresis, followed by silver staining or western blotting; (ii) method 2: this method was used for immunoprecipitations of less-soluble proteins than are possible using method 1. AKAP3 protein was found to be very insoluble and could not be well dissolved or immunoprecipitated by the lysis buffer above. Here, we used a novel modified immunoprecipitation strategy for insoluble or less-soluble proteins. Spermatozoa $\left(8 \times 10^{8}\right)$ were resuspended in $4 \mathrm{ml}$ Celis buffer containing the complete protease inhibitor cocktail, but lacking DTT, and then incubated for $0.5-1 \mathrm{~h}$ at $4{ }^{\circ} \mathrm{C}$ on a rocking platform. The suspension was centrifuged at $4{ }^{\circ} \mathrm{C}, 12000 \mathrm{~g}$ in a table-top microfuge for $10 \mathrm{~min}$ to remove debris. The supernatant was transferred to a dialysis cassette with $10-\mathrm{kDa}$ cutoff and dialysed against $0.1 \times$ phosphate-buffered saline (PBS) (one-tenth strength) for $24 \mathrm{~h}$ at $4{ }^{\circ} \mathrm{C}$ with two changes of PBS. The dialysed suspension was centrifuged at $4{ }^{\circ} \mathrm{C}$ and $6000 \mathrm{~g}$ in a table-top microfuge for $10 \mathrm{~min}$ to sediment the precipitated pellet. The suspension was transferred evenly to four $1.5-\mathrm{ml}$ tubes, and immunoprecipitation was performed as described in method 1 . The immunoprecipitate was then retrieved by eluting the agarose pellet with $200 \mu$ l Celis buffer or with $50 \mu \mathrm{l} 2 \times$ Laemmli sample buffer. Protein G-agarose was then removed by centrifugation at $12000 \mathrm{~g}$ for $20 \mathrm{~s}$ at $15-25^{\circ} \mathrm{C}$ in a microfuge. The supernatant was transferred to a fresh tube for $2 \mathrm{D}$ gel electrophoresis.

2D Isoelectric focusing (IEF)-sodium dodecyl sulphate polyacrylamide gel electrophoresis (SDS-PAGE) of human sperm proteins

Human sperm proteins immunoprecipitated by different antibodies were applied as the first electrophoretic dimension after adding $2 \%(\mathrm{v} / \mathrm{v})$ ampholines ( $\mathrm{pH} 3.5-10)$. IEF was performed with a Protean IEF Cell (Bio-Rad). Nonlinear strips $(11 \mathrm{~cm}, \mathrm{pH} 3-10)$ were rehydrated at $50 \mathrm{~V}$ for at least $12 \mathrm{~h}$ at a sample loading volume of $200 \mu \mathrm{l}$. IEF was then performed using a linear ramp to $8000 \mathrm{~V}$ for a total of $30000 \mathrm{Vh}$. The current was limited to $50 \mathrm{~mA}$ per strip, and the temperature was maintained at $20^{\circ} \mathrm{C}$. For SDS-PAGE, the IPG strips were incubated for $20 \mathrm{~min}$ in equilibration buffer containing $37.5 \mathrm{mmol} \mathrm{l}^{-1}$ Tris- $\mathrm{HCl}$ $(\mathrm{pH} 8.8), 6 \mathrm{~mol} \mathrm{l}^{-1}$ urea, 4\% (w/v) SDS, 20\% (v/v) glycerol and $100 \mathrm{mmol}^{-1}$ DTT. Equilibrated IPG strips were then transferred for the second dimension SDS-PAGE onto Criterion 4-15\% linear gradient gels. Electrophoresis was carried out at room temperature.

\section{Immunoblotting}

Proteins were transferred from unstained gels to polyvinylidine fluoride membranes with a Bio-Rad Trans Blot Electrophoretic Transfer Cell according to the manufacturer's instructions. Membranes were blocked with 5\% (w/v) non-fat milk in PBS for $1 \mathrm{~h}$ at room temperature, washed three times with PBS-Tween (0.05\% (v/v) Tween-20 in PBS), and then incubated overnight at $4{ }^{\circ} \mathrm{C}$ with $15 \mathrm{ml}$ of the previously determined working dilution of rat pre-immune and immune sera (anti-CABYR-A serum in 1:3000; anti-AKAP3 serum in 1:2000). After being washed again, the membranes were incubated with horseradish peroxidaseconjugated goat anti-rat immunoglobulins (Sigma-Aldrich). The signal was detected by enhanced chemiluminescence (Amersham Pharmacia Biotech) or developed with 3,3',5,5'-tetramethylbenzidine (Kirkegaard and Perry Labs, Gaithersburg, MD, USA). Then each experiment was performed for three additional times and each blot was probed with specific antibody or a control immunoglobulin G to immunoprecipitate sperm proteins for exactly the same time.

\section{Tandem mass spectrometry peptide sequencing}

Potential co-immunoprecipitating positive spots were cut out of the $2 \mathrm{D}$ gel and microsequenced by tandem mass spectrometry at the W.M. Keck Biomedical Mass Spectrometry Laboratory of the University of Virginia. Briefly, spots were digested in-gel by trypsin overnight at $37{ }^{\circ} \mathrm{C}$ before analysis. The data were analysed by comparison with a database, the Sequest search algorithm against the NCBI non-redundant database.

\section{Yeast two-hybrid assay}

Vectors, yeast and major reagents were supplied as part of the Matchmaker Gal4 Two-Hybrid System 3. All gene segments used were obtained by PCR using $0.2 \mathrm{ng}$ cloned human testicular marathonready cDNA as the template. Detailed information with respect to 
the design of primers is shown in Table 1. The full-length open reading frame of CR-A (49-1527), the deletion construct of CR-A without the RII-like domain (199-1527) (TCR-A) and the full-length open reading frame of CR-B (1546-2142) were cloned into pGADT7. The full-length open-reading frame of AKAP3 (230-2788) and Ropporin (1-636) were cloned into pGBKT7 vectors using standard cloning procedures. The yeast strain AH109 was simultaneously cotransformed with two recombinant plasmids having different selection markers using LiAc-mediated yeast transformation, as described in the Yeast Protocols Handbook (PT3024-1; Clontech). In this two-hybrid system, GAL4 binding domain binds to the GAL upstream activating sequence, and if the fusion proteins interact, the activating domain is brought into proximity with the promoters of four reporter genes (HIS3, ADE2, MEL1 and lacZ), thereby activating transcription and permitting growth on selection medium (His- and Ade-) and the expression of $\alpha$-galactosidase (MEL1 product) and $\beta$-galactosidase (lac $Z$ product). Cotransformed yeast cells were isolated by growth on SD/-Leu/-Trp plates at $30{ }^{\circ} \mathrm{C}$ for 3 days. For high stringency or medium stringency selection, cells were then transferred to SD/-Ade/-His/-Leu/-Trp or SD/-His/-Leu/-Trp plates, supplemented with $20 \mathrm{~g} \mathrm{ml}^{-1} \mathrm{X}$-Gal (5-bromo-4-chloro-3-indolyl- $\alpha$-D-galactopyranoside), and allowed to grow at $30{ }^{\circ} \mathrm{C}$ for $3-5$ days to select for colonies that expressed interacting proteins.

\section{Quantitative $\alpha$-galactosidase assay}

$\mathrm{SD} /$-Leu/-Trp or SD/-His/-Leu/-Trp cultures were inoculated with a single, fresh yeast colony and incubated overnight at $30^{\circ} \mathrm{C}$. The absorbance at $600 \mathrm{~nm}$ of culture solution was measured, and the supernatant was harvested by centrifugation at $14000 \mathrm{~g}$ for $2 \mathrm{~min}$. Supernatant of $40 \mu \mathrm{l}$ was combined with $120 \mu \mathrm{l}$ fresh assay buffer (2:1 ratio of $0.5 \mathrm{~mol}^{-1}$ sodium acetate, $\mathrm{pH} 4.5$, to $100 \mathrm{mmol}^{-1} \mathrm{p}$-nitrophenyl $\alpha$-D-galactopyranoside (Sigma-Aldrich) and incubated at $30{ }^{\circ} \mathrm{C}$ for $60 \mathrm{~min}$. The reaction was stopped by adding $840 \mu \mathrm{l} 0.1 \mathrm{~mol} \mathrm{l}^{-1}$ $\mathrm{Na}_{2} \mathrm{CO}_{3}$. The absorbance was measured at $410 \mathrm{~nm}$ in a $1.5-\mathrm{ml}$ cuvette, and $\alpha$-galactosidase units were calculated with the following formula: $[$ milliunits $/(\mathrm{ml} \times$ cell $)]=\mathrm{A} 410 \times 1000 \mu \mathrm{l} \times 1000 /[16.9(\mathrm{ml} / \mu \mathrm{mol}) \times 60 \mathrm{~min} \times$ $40 \mu \mathrm{l} \times$ A600] (Yeast Protocols Handbook). Each yeast colony was assayed in triplicate.

\section{RESULTS}

Identification of CABYR binding to AKAP3 in human spermatozoa by co-immunoprecipitation

To determine the location of AKAP3 on the 2D gels, the gels of human sperm Celis extracts were probed with AKAP3 polyclonal antibody (Figure 1a) or treated with pre-immune serum (Figure 1b). These located AKAP3 at $\sim 110 \mathrm{kDa}$. Next, the $2 \mathrm{D}$ gels of human sperm extracts immunoprecipitated with method 2 by anti-CABYR-A poly- clonal antibody (Figure 1c) or pre-immune serum (Figure 1d) were probed with anti-AKAP3 polyclonal antibody. An AKAP3-positive spot indicated by the arrow (Figure 1c) was only present in the human sperm immunoprecipitate by the anti-CABYR-A polyclonal antibody but not in that precipitated with pre-immune serum (Figure 1d). Similarly, the $2 \mathrm{D}$ gels of human sperm proteins immunoprecipitated by the antiCABYR-B polyclonal antibody (Figure 1e), but not the pre-immune serum (Figure 1f), produced an AKAP3-positive spot at the same location. To verify these co-immunoprecipitation results, the role of antibodies as precipitating or labelling was reversed using method 2. The $2 \mathrm{D}$ gels of human sperm proteins immunoprecipitated by anti-AKAP3 polyclonal antibody (Figure 2a) or pre-immune serum (Figure 2b) were probed by anti-CABYR-A polyclonal antibody and showed a CABYRA-positive spot indicated by the arrow in Figure 2a located at the position of the $86-\mathrm{kDa}$ CABYR-A variant. Additionally, the $2 \mathrm{D}$ gels of human sperm extracts immunoprecipitated with the anti-AKAP3 polyclonal antibody (Figure $2 \mathrm{c}$ ) or pre-immune serum (Figure $2 \mathrm{~d}$ ) probed by the anti-CABYR-B polyclonal antibody also showed a string of CABYR-B positive spots indicated by the arrow in Figure $2 \mathrm{c}$.

\section{Identification of CABYR binding with Ropporin}

CABYR is a soluble protein. It can be partly dissolved in gentle detergents such as NP-40, Triton-X 100 and Tween 20, though the majority of CABYR is present in the insoluble fraction (data not shown). Therefore, the regular standard co-immunoprecipitation with the gentle detergent was also employed to identify the potential binding partners of CABYR. Here the strategy is to examine silver-stained gels of human sperm extracts immunoprecipitated by the anti-CABYR-A polyclonal antibody (Figure 3a) and pre-immune sera (Figure $3 \mathrm{~b}$ ) and to identify the unique spots identified in the gel by anti-CABYR-A polyclonal antibody by microsequencing in order to identify the potential binding proteins of CABYR. The candidate spots indicated by the arrows (Figure 3a) were removed for mass spectrometric analysis. The peptide sequences and corresponding proteins are shown in Table 2.

Further analysis of these mass spectrometric results indicated that the origin of the inter-alpha-inhibitor $\mathrm{H} 4$ heavy chain is the rat, and careful observation of the silver-stained gel showed that spot 1 was also found (faintly) in the pre-immune control. Ropporin and HSP90AA1 protein are the most likely candidates to be the binding partners of CABYR. Compared with HSP90AA1 of spot 2, Ropporin of spot 3 is abundant, clear and reproducible. These experiments were performed at least three times. The silver stain gel shown here is a representative example.

Verification of CABYR binding to AKAP3 and Ropporin in human spermatozoa by the yeast two-hybrid system

On the basis of the potential functional domains in CABYR, ${ }^{25}$ three different $C A B Y R$ gene segments were used for the construction of

Table 1 Construction of Gal4 fusions of human genes for yeast two-hybrid assay

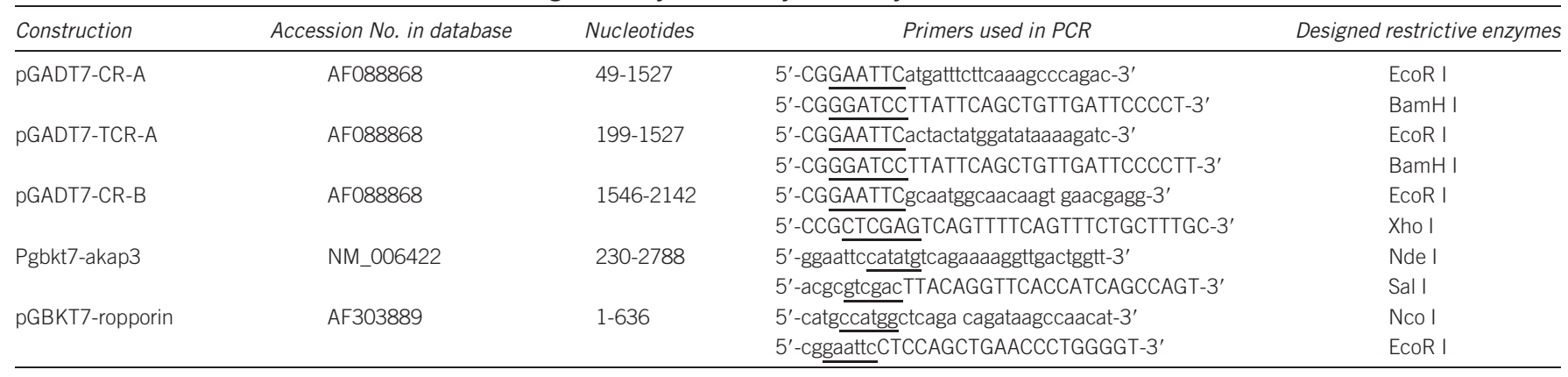



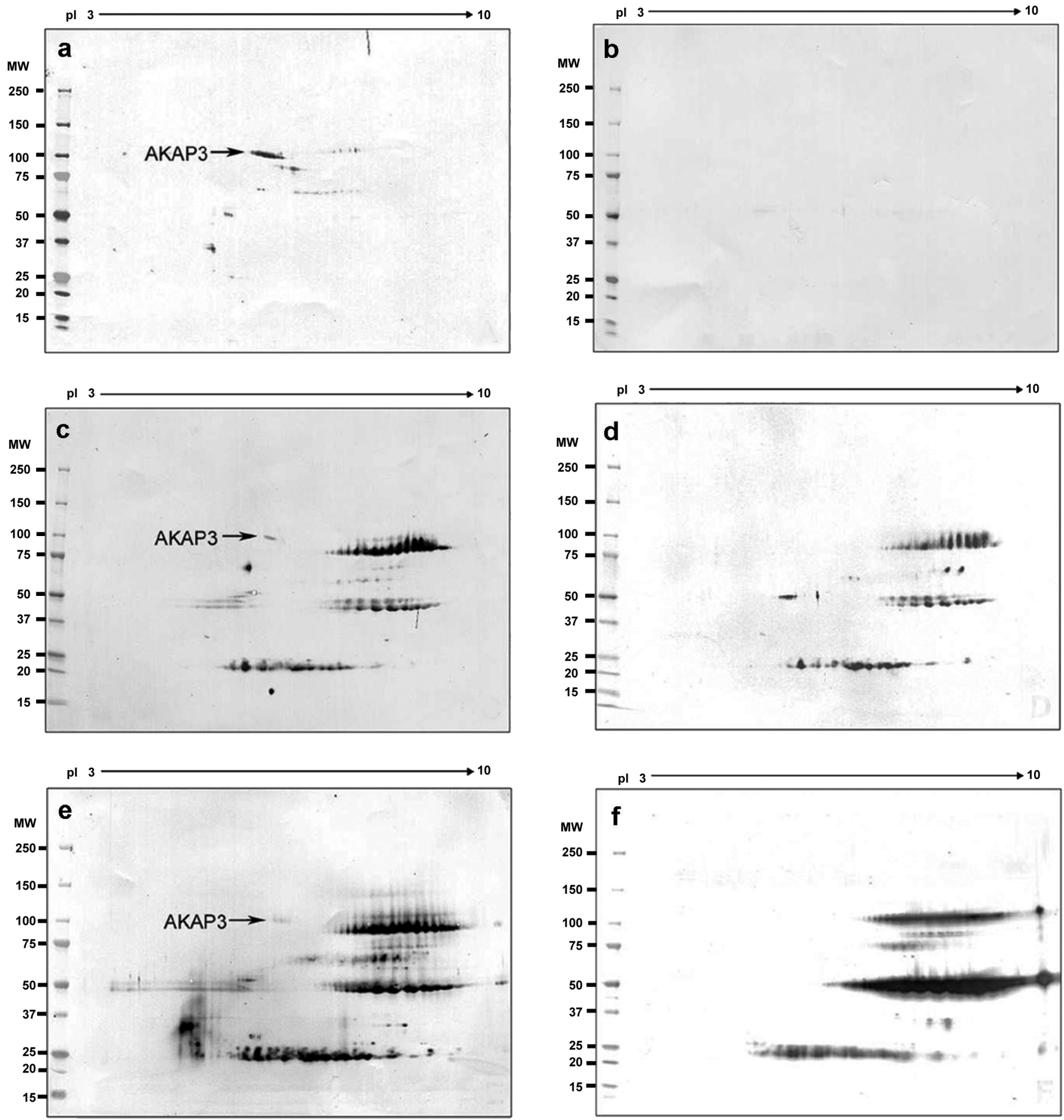

Figure 1 AKAP3 was immunoprecipitated with CABYR by anti-CABYR-A or anti-CABYR-B antibody. (a, b) 2D gels of human sperm extracts probed by anti-AKAP3 polyclonal antibody (a) or pre-immune serum (b). The spot located at $110 \mathrm{kDa}$ with pl 7.0 is the AKAP3. (c, d) 2D gels of human sperm immunoprecipitated by anti-CABYR-A polyclonal antibody (c) or pre-immune serum (d) probed by the anti-AKAP3 polyclonal antibody. The spot indicated by the arrow (c) is the coimmunoprecipitated AKAP3. (e, f) 2D Western blots of human sperm extracts immunoprecipitated by anti-CABYR-B polyclonal antibody (e) or pre-immune serum (f) probed by the anti-AKAP3 polyclonal antibody. The spot indicated by an arrow (e) is the co-immunoprecipitated AKAP3. AKAP, A kinase-anchoring protein; CABYR, calcium-binding tyrosine phosphorylation-regulated protein; 2D, two-dimensional; MW, molecular weight; pl, isoelectric point.

recombinant pGADT7 to verify the interaction between CABYR and AKAP3 or Ropporin and to further examine the role of the RII-like domain of CABYR in binding to AKAP3 or Ropporin (Figure 4). Fulllength open reading frames of AKAP3 and Ropporin were used for the construction of recombinant pGBKT7. The ability of the cotransformants to grow on selective SD/-Leu/-Trp plates (Figure 5a and c) and SD/-Ade/-His/-Leu/-Trp plates (Figure 5b) or on SD/-His/Leu/-Trp plates (Figure 5d) was analysed. The results showed that 

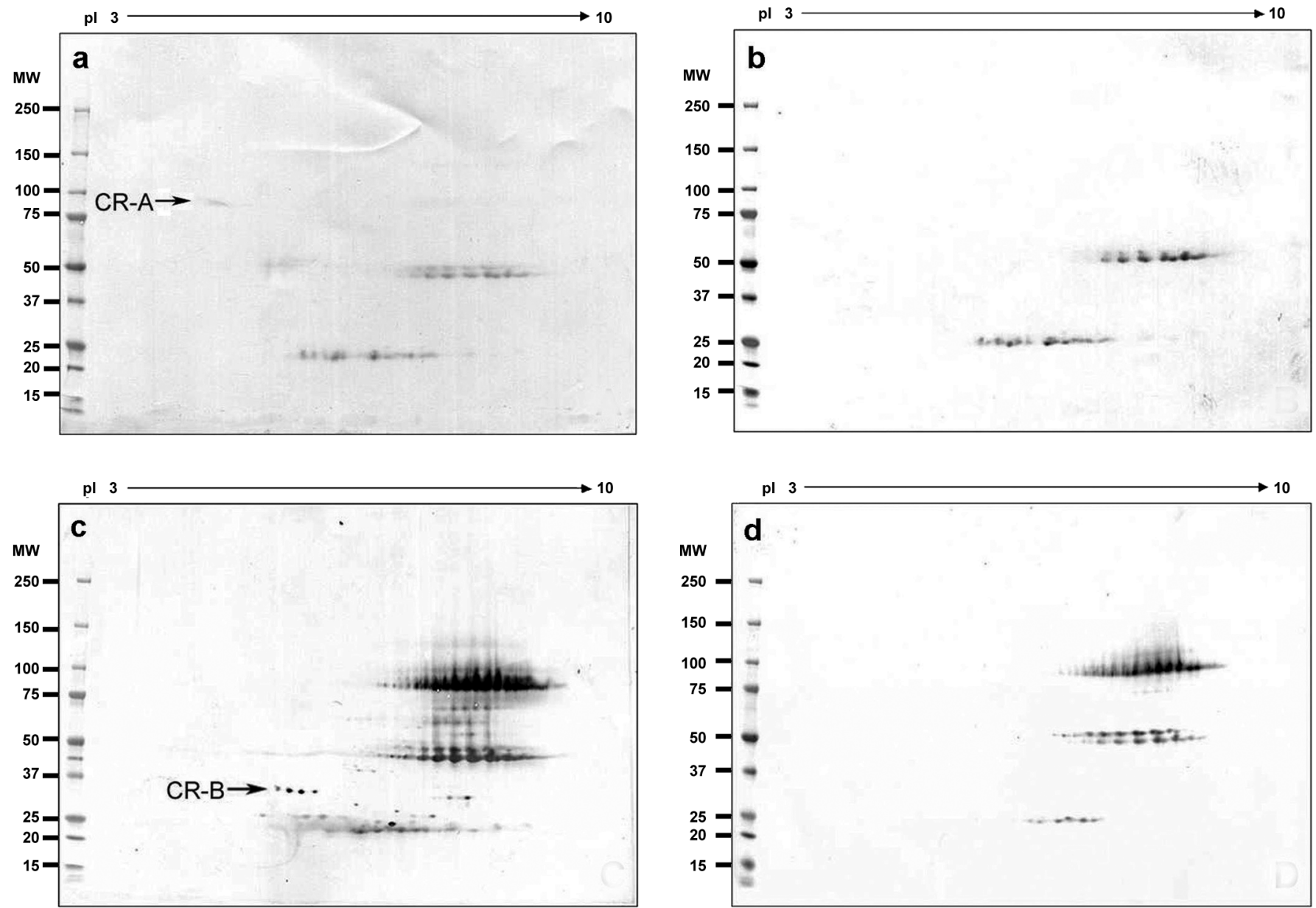

Figure 2 The 86-kDa CABYR-A-only variant and CABYR-B-containing variants were co-immunoprecipitated with AKAP3 by anti-AKAP3 antibody. (a, b) 2D gels of human sperm extracts immunoprecipitated with anti-AKAP3 polyclonal antibody (a) and pre-immune serum (b) probed by the anti-CABYR-A polyclonal antibody. The spot indicated by an arrow (a) is the co-immunoprecipitated 86-kDa CABYR-A-only variant. (c, d) 2D gels of human sperm extracts immunoprecipitated with anti-AKAP3 polyclonal antibody (c) and pre-immune serum (d) probed by the anti-CABYR-B polyclonal antibody. The spot indicated by an arrow (c) is the coimmunoprecipitated CABYR-B-containing variant. AKAP, A kinase-anchoring protein; CABYR, calcium-binding tyrosine phosphorylation-regulated protein; MW, molecular weight; pl, isoelectric point.
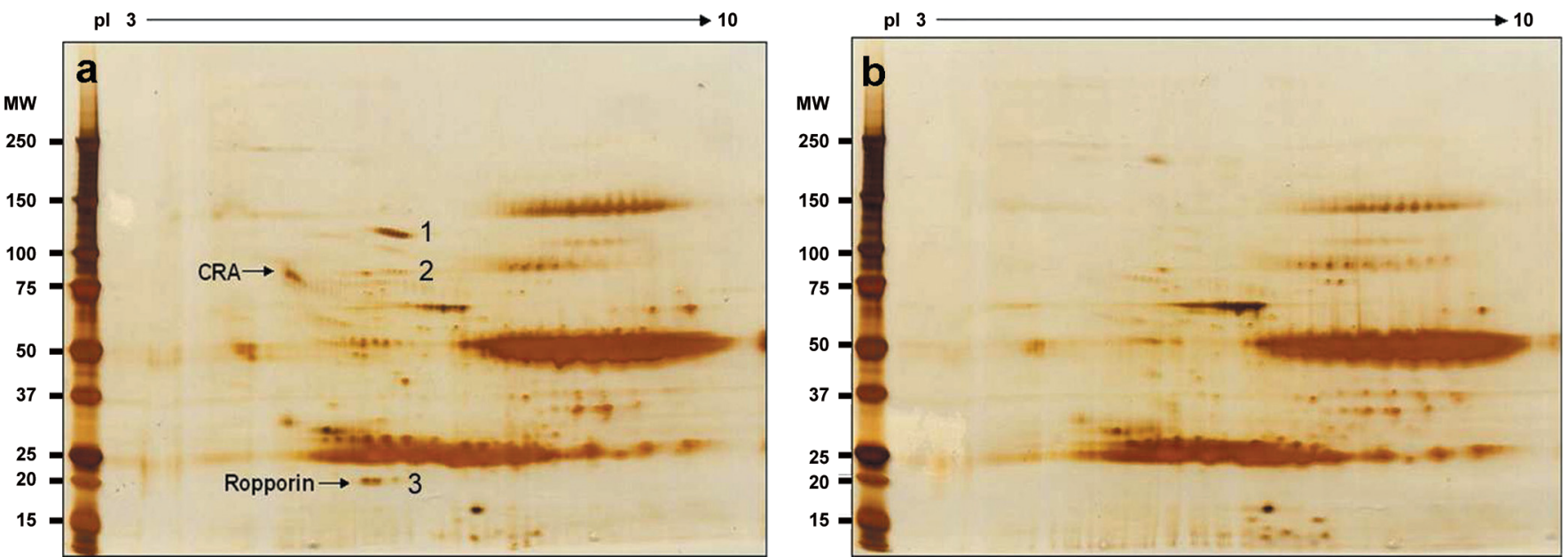

Figure 3 2D silver stain gels of human sperm extracts immunoprecipitated by rat anti-CABYR-A polyclonal antibody (a) and pre-immune serum (b). Three potential positive spots were removed from gels and microsequenced by mass spectrometry. Spot 1 is inter-alpha-inhibitor H4 heavy chain [Rattus norvegicus] (accession no. AAH89806.1); it should come from the rat antiserum; spot 2 contains inter-alpha-inhibitor H4 heavy chain [Rattus norvegicus] and HSP90AA1 protein (accession no. AAH23006.2) sequences; Spot 3 is human Ropporin (accession no. AAG27712.2.). The CABYR-A spot is indicated by the upper arrow. CABYR, calcium-binding tyrosine phosphorylation-regulated protein; 2D, two-dimensional; HSP90, heat-shock protein 90; MW, molecular weight; pl, isoelectric point. 
Table 2 Peptide sequences' corresponding proteins found by mass spectrometry

\begin{tabular}{|c|c|c|c|c|}
\hline $\begin{array}{l}\text { No. of } \\
\text { spots }\end{array}$ & $\begin{array}{l}\text { Accession no. } \\
\text { in database }\end{array}$ & Name of the proteins & Source of species & MS peptides \\
\hline 1 & АAH89806.1 & $\begin{array}{l}\text { Inter-alpha-inhibitor } \\
\text { H4 heavy chain }\end{array}$ & Rattus norvegicus & $\begin{array}{l}\text { FAHTVVTSR } \\
\text { ADTVQEATFQVELPR } \\
\text { GESAGLVK } \\
\text { KTEQFEVSVNVAPGSK } \\
\text { RLGMYELLLKVRPEQLVK } \\
\text { AHIQFKPTLSQQRKSQNEQDTVLDGDFTVR } \\
\text { NVLFVIDK } \\
\text { EALIK } \\
\text { AVDYASKIPAQGGTNINKAVLSAVELLDKSNQAELLPSK } \\
\text { LALDNGGLAR } \\
\text { YNFQHHFKGSEMVVAGKLRDQGPDVLLAK } \\
\text { EFQGPKYIFHNFMERLWALLTIQQQLEQR } \\
\text { YNFVTPLTHMVVTK } \\
\text { LLTSR } \\
\text { LGDGLVGSRQYMPPPGLPGPPGLPGPPGPPGHPHFASSIDYGR } \\
\text { VVEQEGTTPEESPNPDHPRAPTIILPLPGSGVDQLCVDILHSEKPMKLFVDINQGLEVVGK } \\
\text { KTLFSVLPGLK } \\
\text { TGLLQLSGPDKVTISLLSLDDPQR } \\
\text { VLGIDYPATR } \\
\text { LSYQDGFPGTEISCWTVK }\end{array}$ \\
\hline 2 & АAH23006.2 & HSP90AA1 protein & Homo sapiens & $\begin{array}{l}\text { ADLINNLGTIAK } \\
\text { EDQTEYLEER } \\
\text { YIDQEELNK } \\
\text { NPDDITNEEYGEFYK } \\
\text { HFSVEGQLEFRALLFVPR } \\
\text { HIYYITGETKDQVANSAFVER }\end{array}$ \\
\hline & ААН89806.1 & $\begin{array}{l}\text { Inter-alpha-inhibitor } \\
\text { H4 heavy chain }\end{array}$ & Rattus norvegicus & $\begin{array}{l}\text { FAHTVVTSR } \\
\text { ADTVQEATFQVELPR } \\
\text { QYTAAVGRGESAGLVK } \\
\text { KTEQFEVSVNVAPGSK } \\
\text { RLGMYEL } \\
\text { LLKVRPEQLVK } \\
\text { AHIQFKPTLSQQRKSQNEQDTVLDGDFTVR } \\
\text { NVLFVIDK } \\
\text { EALIK } \\
\text { IPAQGGTNINKAVLSAVELLDKSNQAELLPSK } \\
\text { LALDNGGLAR } \\
\text { YNFQHHFKGSEMVVAGKLRDQGPDVLLAK } \\
\text { YIFHNFMER } \\
\text { LGDGLVGSRQYMPPPGLPGPPGLPGPPGPPGHPHFASSIDYGR }\end{array}$ \\
\hline 3 & $\begin{array}{l}\text { AAG27712.2 or } \\
\text { NP_060048.2 }\end{array}$ & Ropporin & Homo sapiens & $\begin{array}{l}\text { AELTPELLKILHSQVAGR } \\
\text { AEELAQMWKVVNLPTDLFNSVMNVGRFTEEIEWLK } \\
\text { IPFSTFQFLYTYIAKVDGEISASHVSR }\end{array}$ \\
\hline
\end{tabular}

Abbreviation: MS, mass spectrometry.

transformants with CR-A+AKAP3 grew normally in selective SD/Ade/-His/-Leu/-Trp medium, as did the positive control. More importantly, when X- $\alpha$-gal was spread on top of the medium, the transformants gradually became blue just like the positive control after 3-5 days in culture at $30^{\circ} \mathrm{C}$ (Figure 5b1). This result indicated that all reporter genes were activated by the interaction between CABYR-A and AKAP3. By contrast, the transformant with TCR-A+AKAP3 (Figure 5b2) could not grow in this medium. The transformants with CR-B + AKAP3 (Figure 5b3) also grew in the selective SD/-Ade/-His/Leu/-Trp medium, but their growth was slower, and the cells were more dispersed. This cotransformant also turned a blue colour. The colour was not as intense as that of the positive control, suggesting that there is a relatively weak interaction between CABYR-B and AKAP3 compared with that of CABYR-A and AKAP3. No cotransformants with CR-A+Ropporin, TCR-A+Ropporin or CR-B + Ropporin could grow in high-stringency SD/-Ade/-His/-Leu/-Trp medium, but the first two could grow in medium-stringency SD/-His/-Leu/-Trp (Figure $5 \mathrm{~d} 1$ and 2) medium, as could the positive control (Figure 5d4). However, the transformant with CR-B+Ropporin (Figure 5d3) did not grow in the same way as the negative control (Figure $5 \mathrm{~d} 5$ ). These results suggest that there is a small and weak interaction between CABYR-A and Ropporin as well as between TCABYR-A and Ropporin, but not between CABYR-B and Ropporin. Unlike in the interaction between CABYR-A and AKAP3, the deletion of the CR-A RII domain did have an effect on, but did not totally abolish, the interaction between CABYR-A and Ropporin.

The quantitative $\alpha$-galactosidase assay also confirmed the findings above. The $\alpha$-galactosidase activity of the transformant bearing CR$\mathrm{A}+\mathrm{AKAP} 3$ was higher than that of P53+SV40 large T (positive control). By contrast, the transformant bearing TCR-A+AKAP3, in which 


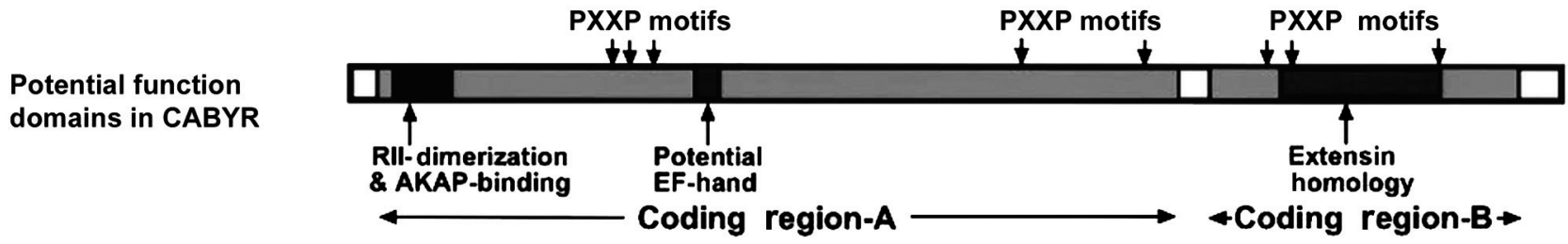

Full-length CR-A

Truncated CR-A deleted the RII domain (TCR-A)

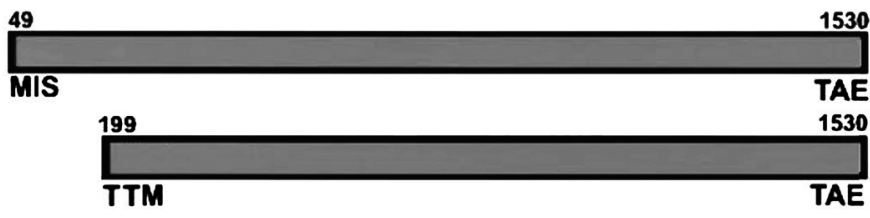

Full-length CR-B

Figure 4 A schematic map of CABYR adopted with permission from Naaby-Hansen et al. ${ }^{25}$ illustrates the positions of different hypothetical domains and the CABYR gene segments used for the construction of transformants. The numbers refer to the aa positions. aa, amino acid; CABYR, calcium-binding tyrosine phosphorylationregulated protein; $\mathrm{CR}$, coding region.

the TCR-A construct deleted the RII-like domain, had very low activity similar to that of the P53+Lam C (negative control). These results strongly indicate that the interaction between CABYR-A and AKAP3 is mediated by the RII-like domain. The transformants bearing CR$\mathrm{B}+\mathrm{AKAP} 3$, CR-A+Ropporin and TCR-A+Ropporin exhibit distinctly lower activities than those of the positive control but clearly higher activities than those of the negative control, supporting the finding above that there is a relatively weak interaction between CABYR-B and AKAP3 and between CABYR-A and Ropporin or between TCABYR-A and Ropporin. Because the transformant bearing TCR-A+Ropporin had a much lower activity than did the one bearing CR-A+Ropporin, the weak interaction between CABYR-A and Ropporin may be mediated not only by the RII domain but also by some other domain. The transformant bearing CR-B+Ropporin had very low $\alpha$-galactosidase activity, similar to that of P53+Lam C (negative control) (Figure 5e). The data from the quantitative $\alpha$-galactosidase assay here are consistent with the findings from the growth assay of the transformants on selective medium. The interaction between CABYR and AKAP3 or Ropporin is summarized in Figure $5 \mathrm{f}$.

\section{DISCUSSION}

The interaction between CABYR and AKAP3

Previous bioinformatic analysis of CABYR suggests that the domain in CABYR-A of CABYR, like that of Ropporin and sperm autoantigenic protein 17 , shares a strong sequence similarity with the conserved RII $\alpha$ domain of protein kinase A. ${ }^{25}$ The binding experiments cannot be performed with native AKAP3 because this protein is highly insoluble. ${ }^{28}$ The difficulty of identifying the protein-protein interaction in the FS by co-immunoprecipitation lay in the impossibility of dissolving any of the related proteins using the regular lysis buffer. Most of the intrinsic FS proteins, including AKAPs, are rather insoluble proteins. ${ }^{1,34}$ So far, most studies of the interactions between AKAPs and their binding partners have used recombinant transfected cells, such as COS-7, ${ }^{23}$ HEK293, ${ }^{35}$ bacteria $^{12,36}$ or the yeast two-hybrid system. ${ }^{6,22,35}$ Early in the present study, the standard co-immunoprecipitation method was tried but was revealed not to resolve AKAPs or make them immunoprecipitate (data not shown). To dissolve all proteins in sufficient amounts and to identify the potential binding partners of CABYR, a novel co-immunoprecipitation strategy for less insoluble and insoluble proteins was employed and produced sat- isfactory results. The data here showed that at least some of the protein-protein interactions could be maintained in the Celis buffer without DTT and $\beta$-ME and that the interaction could be recovered after the extract was renatured by dialysis against PBS. The present study, in which both co-immunoprecipitation and yeast two-hybrid analysis were used, confirmed that there does exist an interaction between CABYR and AKAP3. Not only was it shown that the native proteins of CABYR and AKAP3 could be co-immunoprecipitated with each other by either one of their polyclonal antibodies, but it was also shown that the recombinant CABYR and AKAP3 proteins significantly interacted in the yeast two-hybrid assay. Here, the cotransformed transformants bearing constructs expressing both recombinant CABYR and AKAP3 grew normally in the selective SD/-Ade/-His/-Leu/-Trp medium, suggesting that the interaction between CABYR and AKAP3 activates the expression of the reporter genes ADE2 and HIS3; the MEL1 reporter gene is also activated as demonstrated by the quantitative $\alpha$-galactosidase assays. This result is not consistent with a previous report showing the interaction between CABYR and AKAPs to be much more limited. ${ }^{29}$ Conversely, the strength of this interaction was much stronger than that between P53 and SV40 large T. Importantly, when one of the constructs expressed the truncated CR-A in which the RII domain had been deleted, the cotransformed AH109 with the construct expressing the full-length AKAP3 could not grow in selective SD/Ade/-His/-Leu/-Trp medium, and the $\alpha$-galactosidase activity of this cotransformant was as low as in the negative control. This provides direct evidence that the interaction between CABYR and scaffolding protein AKAP3 in the FS of human sperm is mediated by the RII domain on the N-terminus of CABYR. Because the anchoring of RII of protein kinase A to AKAP requires RII dimerisation, it could be that the dimerisation of CABYR between different variants is necessary for mediating its binding to AKAP3. As CABYR is a calcium-binding, tyrosine- as well as serine- or threonine-phosphorylated protein, its binding to AKAP3 may reflect a functional need for $\mathrm{Ca}^{2+}$ signalling transduction, and it may play a role in motility at sites along the FS.

\section{The interaction between CABYR and Ropporin}

The ultrastructural location of Ropporin is mainly on the inner surface of the FS. ${ }^{19}$ This specific location is close to that of CABYR, which is present within the FS, including the surface of the longitudinal column 

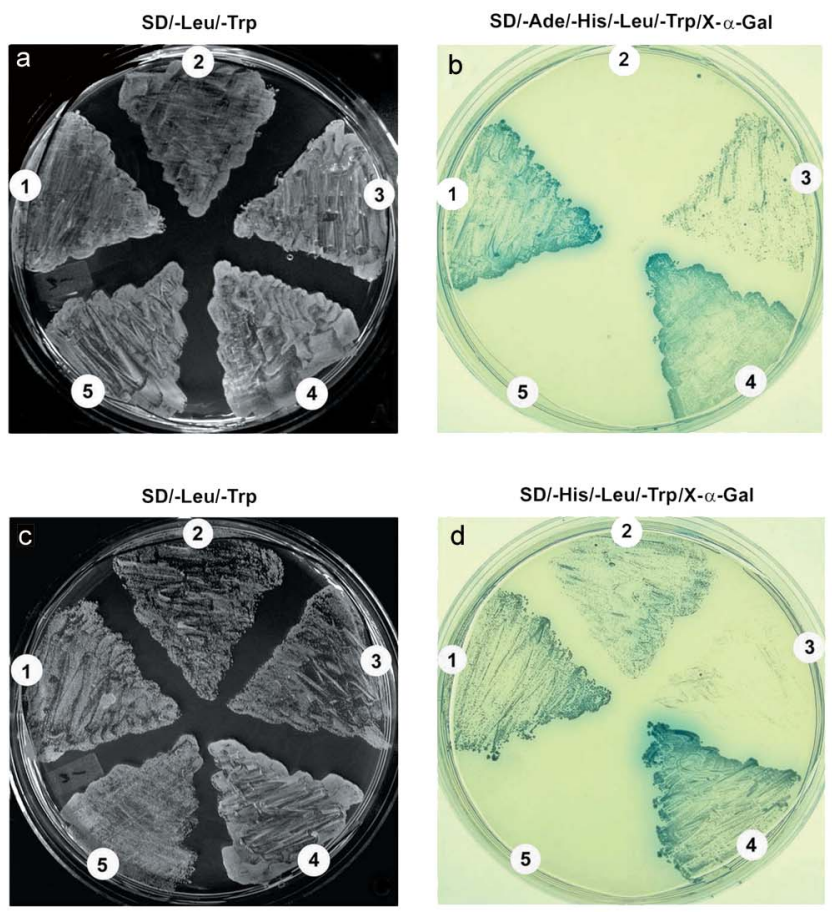

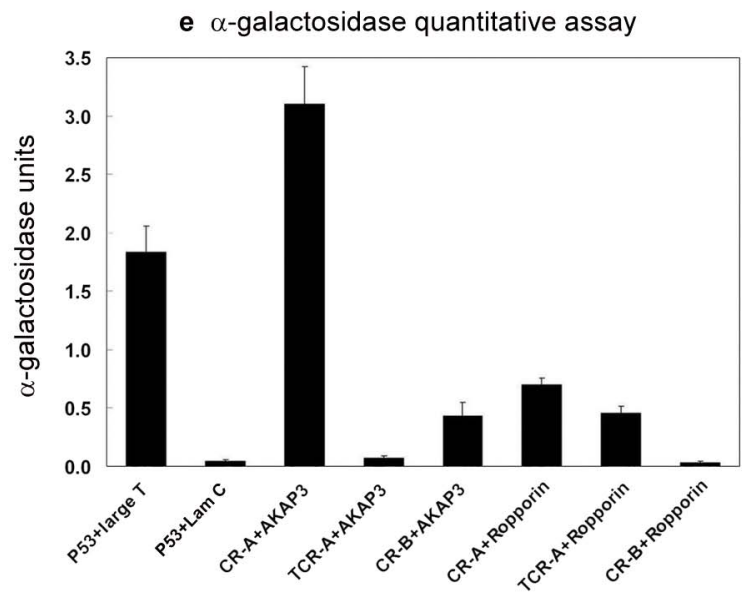

f Summary of the interactions

\begin{tabular}{ccc}
\hline Gene segments & AKAP3 & Ropporin \\
\hline hCR-A & +++ & + \\
hTCR-A & - & + \\
hCR-B & + & \\
\hline
\end{tabular}

Figure 5 Verification of the interaction between CABYR and AKAP3 or Ropporin by the yeast two-hybrid assay. The yeast strain AH109 was cotransformed with pGADT7-hCR-A, pGADT7-hTCR-A or pGADT7-hCR-B and pGBKT7-AKAP3, pGBKT7-Ropporin (cotransformed with pGADT7-T and pGBKT7-53 as a positive control; pGADT7-T and pGBKT7-Lam as a negative control). The abilities of the transformants to grow on selective SD/-Leu/-Trp plates (a, c), SD/-Ade/-His/ -Leu/-Trp plates (b) or SD/-His/-Leu/-Trp plates (d) were analysed. (a, b) The analysis of the interaction between CABYR and AKAP3: 1. CR-A+AKAP3; 2. TCRA+AKAP3; 3. CR-B +AKAP3; 4. p53+ Large T (positive control); 5. p53+Lam C (negative control). (c, d) The analysis of the interaction between CABYR and Ropporin: 1. CR-A + Ropporin; 2. TCR-A+Ropporin; 3. CR-B + Ropporin; 4. p53+Large T (positive control); 5. p53+Lam C (negative control). (e) $\alpha$-galactosidase quantitative data are plotted with error bars corresponding to three separate experiments. The $\alpha$-galactosidase activity of the transformant bearing CR-A+AKAP3 is much higher than that the transformant bearing the P53+SV40 large T. TCR-A+AKAP3 has very low $\alpha$-galactosidase activity similar to that of p53+Lam C. (f) A summary of the interaction between CABYR and AKAP3 or Ropporin. AKAP, A kinase-anchoring protein; CABYR, calcium-binding tyrosine phosphorylation-regulated protein; CR, coding region; TCR, truncated coding region.

and ribs, as well as over electron-dense material lying between the FS and the outer dense fibre. ${ }^{25}$ Biochemical characterisation has shown that Ropporin, unlike the intrinsic components of the FS, which are known to be resistant to $6 \mathrm{~mol}^{-1}$ urea, ${ }^{30}$ is present in both Triton $\mathrm{X}-100$-soluble and -insoluble fractions and was almost completely dissolved in $4 \mathrm{~mol}^{-1}$ urea, suggesting that Ropporin is essentially a soluble protein and binds to the FS. Similarly, our experiments showed that CABYR is present in both Triton X-100-soluble and -insoluble fractions, and so it should be a soluble protein that binds to the surface of FS as does Ropporin. Furthermore, the N-terminal sequences of both CABYR and Ropporin are highly homologous to the dimerisation motif of RII $\alpha$ of protein kinase A. On the basis of the similar pattern of localisation, biochemical characteristics and molecular structure of CABYR and Ropporin, we primarily pursued evidence for interaction between CABYR and AKAP3, as found for the interaction between Ropporin and $\mathrm{AKAP} 3,{ }^{22}$ and revealed an interaction between CABYR and AKAP3. The present study showed that Ropporin was co-immunoprecipitated with CABYR by an antiCABYR-A polyclonal antibody by regular co-immunoprecipitation methods involving gentle lysis. Reproducible experiments showed that a short string of spots with molecular weights of about $20 \mathrm{kDa}$ and isoelectric point (pI) 5-6 were always present on the silver stain gels of an anti-CABYR-A polyclonal antibody immunoprecipitate, and microsequencing indicated that they were Ropporin. Proteins from mouse sperm immunoprecipitated with anti-mouse CABYR-A polyclonal antibody also have the same Ropporin spots, identified by mass spectrometry. ${ }^{37}$ A yeast two-hybrid assay in which constructs expressed the full-length Ropporin protein, different CABYR segments, truncated CABYR-A in which the RII domain had been deleted or CABYR-B confirmed these findings. Unlike the findings for AKAP3 and CABYR, both full-length and truncated CABYR-A could bind to Ropporin. The interaction between CABYR-A and Ropporin was weaker than that between CABYR-A and AKAP3, and CABYR-B did not interact with Ropporin. Obviously, CABYR not only dimerizes with its different variants but also forms heterodimers or oligomers with its binding partner Ropporin. This result is also supported indirectly by the finding that Ropporin forms a heterodimer with sperm autoantigenic protein 17 in vitro. ${ }^{19}$

The yeast two-hybrid assay not only verified the interactions between CABYR and AKAP3 or Ropporin, but also indicated that the RII-domain of CABYR is critical for its binding with AKAP3 but may not be the only domain for its binding to Ropporin. CABYR-B of CABYR also participates in the binding to AKAP3, albeit weakly, but not in the binding to Ropporin. The interaction between CABYR-A and AKAP3 is much stronger than that of P53 and SV40 large T according to the quantitative $\alpha$-galactosidase data.

In summary, direct evidence for the interaction between CABYR and AKAP3 or Ropporin in human spermatozoa was demonstrated by both co-immunoprecipitation and the yeast two-hybrid interaction assay. Deletion of the RII domain abolished the interaction between CABYR and AKAP3 but did not entirely abolish the interaction between CABYR and Ropporin. Thus, CABYR binds to AKAP3 by its RII domain but 
also binds to Ropporin by the RII domain and other domains. These interactions between CABYR and AKAP3 or Ropporin could be involved in the assembly of signalling complexes in the FS.

\section{AUTHOR CONTRIBUTIONS}

YFL and WH contributed equally to this work. YFL and WH conceived the study, designed and carried out the experiments, and drafted the manuscript. AM and YHK prepared the antibodies and participated in part of the co-immunoprecipitation study. LD and KK gave technical support and beneficial advice. CJF participated in the design of the study and helped to draft the manuscript. JCH administered the experiment, participated in its design and coordination, and helped to draft the manuscript. All authors read and approved the final manuscript.

\section{COMPETING FINANCIAL INTERESTS}

The author declare no competing financial interests.

\section{ACKNOWLEDGMENTS}

This work was supported by D43 TW/HD 000654 from the Fogarty International Center, NIH, USA.

1 Eddy EM, Toshimori K, O'Brien DA. Fibrous sheath of mammalian spermatozoa. Microsc Res Tech 2003; 61: 103-15.

2 Fawcett DW. The mammalian spermatozoon. Dev Biol 1975; 44: 394-436.

3 Vijayaraghavan S, Liberty GA, Mohan J, Winfrey VP, Olson GE et al. Isolation and molecular characterization of AKAP110, a novel, sperm-specific protein kinase A-anchoring protein. Mol Endocrinol 1999; 13: 705-17.

4 Mandal A, Naaby-Hansen S, Wolkowicz MJ, Klotz K, Shetty J et al. FSP95, a testisspecific 95-kilodalton FS antigen that undergoes tyrosine phosphorylation in capacitated human spermatozoa. Biol Reprod 1999; 61: 1184-97.

5 Carrera A, Gerton GL, Moss SB. The major FS polypeptide of mouse sperm: structural and functional similarities to the A-kinase anchoring proteins. Dev Biol 1994; 165: 272-84.

6 Brown PR, Miki K, Harper DB, Eddy EM. A-kinase anchoring protein 4 binding proteins in the FS of the sperm flagellum. Biol Reprod 2003; 68: 2241-8

7 Mei X, Singh IS, Erlichman J, Orr GA. Cloning and characterization of a testis-specific, developmentally regulated A-kinase-anchoring protein (TAKAP-80) present on the FS of rat sperm. Eur J Biochem 1997; 246: 425-32.

8 Welch JE, Schatte EC, O'Brien DA, Eddy EM. Expression of a glyceraldehydes 3-phosphate dehydrogenase gene specific to mouse spermatogenic cells. Biol Reprod 1992; 46: 869-78.

9 Bunch DO, Welch JE, Magyar PL, Eddy EM, O'Brien DA. Glyceraldehyde 3-phosphate dehydrogenase-S protein distribution during mouse spermatogenesis. Biol Reprod 1998; 58: 834-41.

10 Mori C, Welch JE, Fulcher KD, O'Brien DA, Eddy EM. Unique hexokinase messenger ribonucleic acids lacking the porin-binding domain are developmentally expressed in mouse spermatogenic cells. Biol Reprod 1993; 49: 191-203.

11 Visconti PE, Olds-Clarke P, Moss SB, Kalab P, Travis AJ et al. Properties and localization of a tyrosine phosphorylated form of hexokinase in mouse sperm. $\mathrm{Mol}$ Reprod Dev 1996; 43: 82-93.

12 Mori C, Nakamura N, Welch JE, Gotoh H, Goulding EH et al. Mouse spermatogenic cellspecific type 1 hexokinase (mHk1-s) transcripts are expressed by alternative splicing from the $m H k 1$ gene and the HK1-S protein is localized mainly in the sperm tail. Mol Reprod Dev 1998; 49: 374-85.

13 Travis AJ, Foster JA, Rosenbaum NA, Visconti PE, Gerton GL et al. Targeting of a germ cell-specific type 1 hexokinase lacking a porin-binding domain to the mitochondria as well as to the head and FS of murine spermatozoa. Mol Biol Cell 1998; 9: 263-76.
14 Hsu HC, Lee YL, Cheng TS, Howng SL, Chang LK et al. Characterization of two nontestis-specific CABYR variants that bind to GSK3b with a proline-rich extensionlike domain. Biochem Biophys Res Commun 2005; 329: 1108-17.

15 Krisfalusi M, Miki K, Magyar PL, O'Brien DA. Multiple glycolytic enzymes are tightly bound to the FS of mouse spermatozoa. Biol Reprod 2006; 75: 270-78.

$16 \mathrm{Kim} \mathrm{YH,} \mathrm{Haidl} \mathrm{G,} \mathrm{Schaefer} \mathrm{M,} \mathrm{Egner} \mathrm{U,} \mathrm{Mandal} \mathrm{A} \mathrm{et} \mathrm{al.} \mathrm{Compartmentalization} \mathrm{of} \mathrm{a}$ unique ADP/ATP carrier protein SFEC (sperm flagellar energy carrier, AAC4) with glycolytic enzymes in the fibrous sheath of the human sperm flagellar principal piece. Dev Biol 2007; 302: 463-76.

17 Fulcher KD, Welch JE, Klapper DG, O'Brien DA, Eddy EM. Identification of a unique mclass gluthathione S-transferase in mouse spermatogenic cells. Mol Reprod Dev 1995; 42: 415-24.

18 Catalano RD, Hillhouse EW, Vlad M. Developmental expression and characterization of FS39, a testis complementary DNA encoding an intermediate filament-related protein of the sperm FS. Biol Reprod 2001; 65: 277-87.

19 Fujita A, Nakamura K, Kato T, Watanabe N, Ishizaki T et al. Ropporin, a sperm-specific binding protein of rhophilin, that is localized in the FS of sperm flagella. J Cell Sci 2000; 113: 103-12.

20 Kong M, Richardson RT, Widgren EE, O'Rand MG. Sequence and localization of the mouse sperm autoantigenic protein, Sp17. Biol Reprod 1995; 53: 579-90.

21 Frayne J, Hall L. A re-evaluation of sperm protein 17 (Sp17) indicates a regulatory role in an A-kinase anchoring protein complex, rather than a unique role in sperm-zona pellucida binding. Reproduction 2002; 124: 767-74.

22 Carr DW, Fujita A, Stentz CL, Liberty GA, Olsen GE et al. Identification of sperm specific proteins that interact with A-kinase anchoring proteins in a manner similar to the type II regulatory subunit of PKA. J Biol Chem 2001; 276: 17332-8.

23 Bajpai M, Fiedler SE, Huang Z, Vijayaraghavan S, Olson GE et al. AKAP3 selectively binds PDE4A isoforms in bovine spermatozoa. Biol Reprod 2006; 74: 109-18.

$24 \mathrm{Li} \mathrm{YF,} \mathrm{He} \mathrm{W,} \mathrm{Jha} \mathrm{KN,} \mathrm{Klotz} \mathrm{K,} \mathrm{Kim} \mathrm{YH} \mathrm{et} \mathrm{al.} \mathrm{FSCB,} \mathrm{a} \mathrm{novel} \mathrm{protein} \mathrm{kinase}$ Aphosphorylated calcium-binding protein, is a CABYR-binding partner involved in late steps of fibrous sheath biogenesis. J Biol Chem 2007; 282: 34104-19.

25 Naaby-Hansen S, Mandal A, Wolkowicz MJ, Sen B, Westbrook VA et al. CABYR, a novel calcium-binding tyrosine phosphorylation-regulated fibrous sheath protein involved in capacitation. Dev Biol 2002; 242: 236-54.

26 Ficarro S, Chertihin O, Westbrook VA, White F, Jayes F et al. Phosphoproteome analysis of capacitated human sperm. Evidence of tyrosine phosphorylation of AKAP 3 and valosin containing protein/P97 during capacitation. J Biol Chem 2003. 278: 11579-89.

27 Fiedler SE, Bajpai M, Carr DW. Identification and characterization of RHOAinteracting proteins in bovine spermatozoa. Biol Reprod 2007; 78: 184-92.

28 Lea IA, Widgren EE, O'Rand MG. Association of sperm protein 17 with A-kinase anchoring protein 3 in flagella. Reprod Biol Endocrinol 2004; 2: 57.

29 Hanlon Newell AE, Fiedler SE, Ruan JM, Pan J, Wang PJ et al. Protein kinase A RII-like (R2D2) proteins exhibit differential localization and AKAP interaction. Cell Motil Cytoskel 2008; 65: 539-552.

$30 \mathrm{Kim}$ YH, Jha KN, Mandal A, Vanage G, Farris E et al. Translation and assembly of CABYR coding region $B$ in fibrous sheath and restriction of calcium binding to coding region A. Dev Biol2005; 286: 46-56

31 Luconi M, Porazzi I, Ferruzzi P, Marchiani S, Forti G et al. Tyrosine phosphorylation of the A kinase anchoring protein 3 (AKAP3) and soluble adenylate cyclase are involved in the increase of human sperm motility by bicarbonate. Biol Reprod 2005; 72: 22-32.

32 World Health Organization. WHO Laboratory Manual for the Examination of Human Semen and Semen-Cervical Mucus Interaction. 3rd ed. Cambridge: Cambridge University Press.

33 Bronson RA, Fusi F. Sperm-oolemmal interaction: role of the Arg-Gly-Asp (RGD) adhesion peptide. Fertil Steril 1990; 54: 527-529.

34 Brito M, Figueroa J, Maldonado EU, Vera JC, Burzio LO. The major component of the rat sperm FS is a phosphoprotein. Gamete Res 1989; 22: 205-17.

35 Carlson CR, Ruppelt A, Taskén K. A kinase anchoring protein (AKAP) interaction and dimerization of the Rla and RIb regulatory subunits of protein kinase $A$ in vivo by the yeast two hybrid system. J Mol Biol 2003; 327: 609-18.

36 Niu J, Vaiskunaite R, Suzuki N, Kozasa T, Carr DW et al. Interaction of heterotrimeric G13 protein with an A-kinase anchoring protein 110 (AKAP110) mediates cAMP independent PKA activation. Curr Biol 2001; 11: 1686-90.

37 Li YF, He W, Kim YH, Mandal A, Digilio L et al. CABYR isoforms expressed in late steps of spermiogenesis bind with AKAPs and ropporin in mouse sperm fibrous sheath. Reprod Biol Endocrinol 2010; 8: 101, doi:10.1186/1477-7827-8-101. 